\title{
NOD2: ACTIVATION DURING BACTERIAL AND VIRAL INFECTIONS, POLYMORPHISMS AND POTENTIAL AS THERAPEUTIC TARGET
}

\author{
Diana Alhelí Domínguez-Martínez ${ }^{1}$, Daniel Núñez-Avellaneda ${ }^{1}$, Carlos Alberto Castañón-SánéheZ ${ }^{2}$
} AND MA ISABEL SALAZAR ${ }^{3 *}$

${ }^{1}$ Laboratorio de Inmunología Celular e Inmunopatogénesis, Departamento de Inmunología, Escuela Nacional de Cieñcias Biológicas, Instituto Politécnico Nacional, Mexico City; ${ }^{2}$ Subdirección de Enseñanza e Investigación, Hospital Regionāal de Alta Especialidad de Oaxaca, San Bartolo Coyotepec, Oax.; ${ }^{3}$ Sección de Inmunovirología, Laboratorio de Virología, Departamento de Microbiología, Escuela Nacional de Ciencias Biológicas, Instituto Politécnico Nacional, Mexico City, Mexico

\section{ABSTRACT}

Nucleotide-binding domain (NBD) leucine-rich repeat (LRR)-containing receptors or NLRs are a family of receptors thatedect both, molecules associated to pathogens and alarmins, and are located mainly in the cytoplasm. NOD2 belongs to the NLLR family and is a dynamic receptor capable of interacting with multiple proteins and modulate immune responses in a stimuli-dependent manner. The experimental evidence shows that interaction between NOD2 structural domains and the effector proteins shape the overall response against bacterial or viral infections. Other reports have focused on the importance of NOD2 not only in infection but also in maintaining tissue homeostasis. However, not only protein interactions relate to function but alsocertain polymorphisms in the gene that encodes NOD2 have been associated with inflammatory diseases, such as Crohn's "disease. Here, we review the importance and general characteristics of NOD2, discussing its participation in infections caused by bacteria and viruses as well as its interaction with other pathogen recognition receptors or effectors to induce antibacterial and antiviral responses. Finally, the role of NOD2 in chronic inflammatory conditions and its potential to be targeted therapeutically are examined.

Key words: NOD2. Inflammasome. Infection. Polymorphisms. Inflammatory disease.

\section{INTRODUCTION}

The repertoire of pathogen recognition receptors (PRRs) in the innate immune system is encoded in the germline. These PRRs are activated either

\author{
Corresponding author: \\ * Ma Isabel Salazar \\ Laboratorio de Virología \\ Departamento de Microbiología \\ Escuela Nacional de Ciencias Biológicas \\ Instituto Politécnico Nacional \\ Prolong. Manuel M. Carpio y Plan de Ayala, s/n \\ Col. Santo Tomás \\ C.P. 11340, Ciudad de México, México \\ E-mail: isalazarsan@yahoo.com
}

by evolutionarily conserved pathogen-assocjated molecular patterns (PAMPs) or by endogenous damage-associated molecular patterns (DAMPs). PAMPs are molecules present in pathogens; in bactería, they are found in the cell wall, flagella, lipoprotei $\bar{B}$, and
Received for publication: 08-07-2017

Approved for publication: 20-10-2017 doi: $10.24875 /$ RIC.17002327 
Figure 1. PRRs that participate in pathogen recognition and immune response. Pathogen recognition receptors (PRRs) are custered into five families depending on their structural domains. Toll-like receptors (TLRs) are located in cell membrane and endosomes; they recognize a broad range of pathogens through their leucine rich-region (LRR) and signal by toll/interleukine- 1 receptor domains. Retinoic acid-inducible gene-l-like receptors-like receptors (RLRs), such as RIG-I and MDA-5, are located in the cyfoplasm and recognize motifs from pathogens such as dsRNA through its helicase domains (Hel-1 and Hel-2). C-type lectin receptors (CLRs) are transmembranal and include dendritic cell-specific intercellular adhesion molecule-3-grabbing non-integrin (DC-SIGN) and C-type lectin domain family 5 member A (CLEC5a); these identify carbohydrates in a broad number of pathogens. ALRs include molecules such as absent in melanoma 2 (AIM2) are cytoplasmic and recognize mostly foreign dsDNA. Finally, the NLRs aremostly located in cytoplasm and mitochondria, and contain three domains, a variable $\mathrm{N}$-terminal characterized by interacting withseveral effectors, a central nucleotide-binding domain (NBD), and most NLRs have a C-terminal LRR domain.

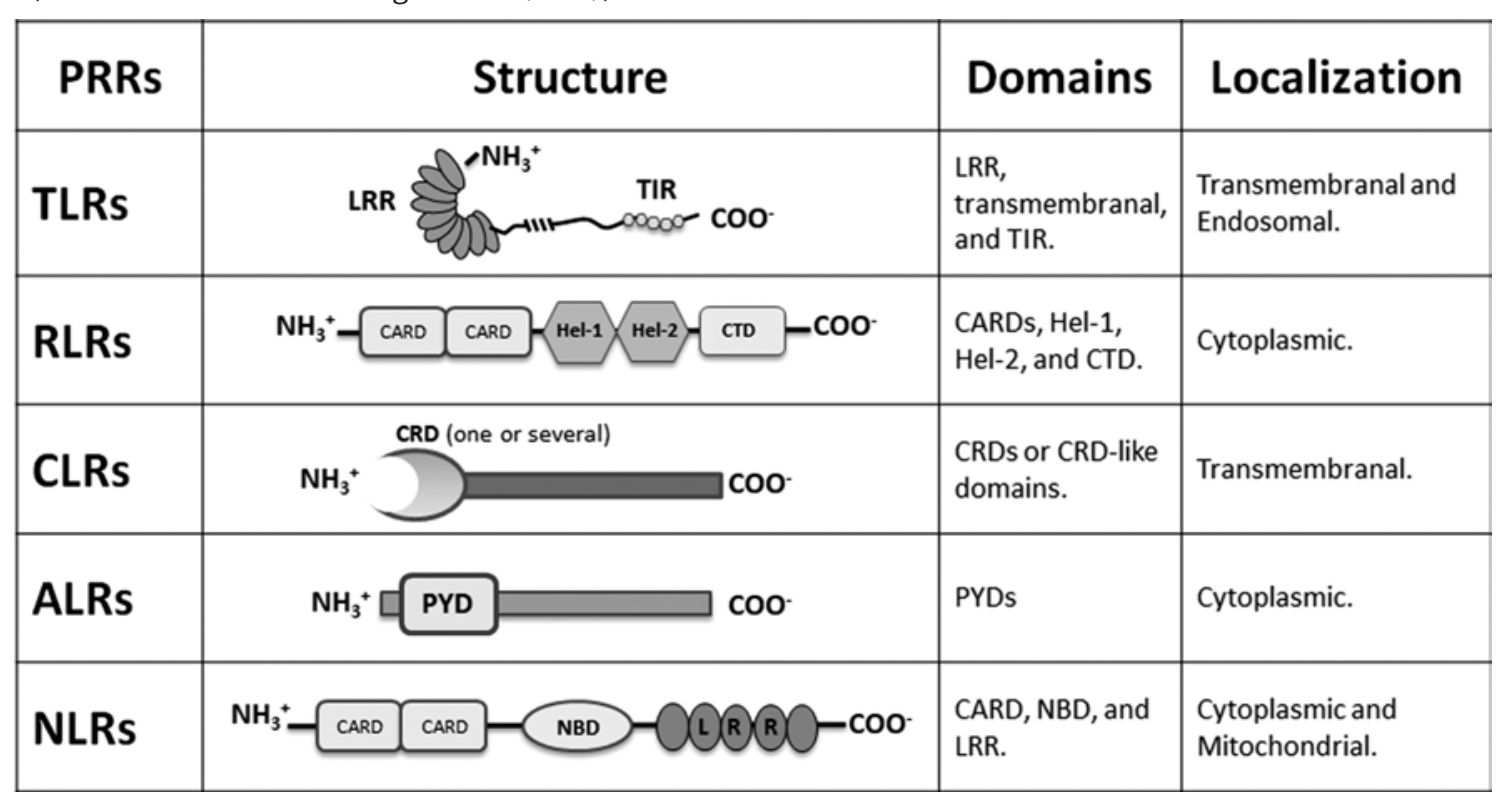

nucleic acids. Furthermore, some cell wall components in fungi, as well as nucleic acids and proteins in viruses, are recognized as PAMPs' ${ }^{1}$. Unlike PAMPs, DAMPs are endogenous molecules released from cells under stress damage; these events are capable of initiating an inflammatory process. Some well-characterized DAMPs include DNA-binding proteins such as high-mobility group B1 proteins, heat-shock proteins, extracellular adenosine triphosphate (ATP), and uric acid crystals, among others.

PRRs are grouped into five families according to their structural domains (Fig. 1): (1) Toll-like receptors (TLRs), (2) retinoic acid-inducible gene-l-like receptors (RLRs), (3) C-type lectin receptors (CLRs), (4) absent in melanoma 2 (AIM2)-like receptors (ALRs), and (5) NOD-like receptors (NLRs) ${ }^{2,3}$. Following is a brief description of these PRRs.

TLRs are transmembrane glycoproteins that express an $\mathrm{N}$-terminal ectodomain containing leucine-rich repeats (LRRs). Toll/interleukin-1 receptor (TIR) domain at their $\mathrm{C}$-terminus allows signal transduction ${ }^{4}$.
TLR interaction with its ligand enables TIR domain dimerization and triggers signal transduction through TIR-domain-containing adapter-inducing interferon- $\beta$ or myeloid differentiation factor 88 (MyD88) $)^{4,5}$. This signaling activates the nuclear factor $\kappa B(N F-\kappa B)$ and induces the transcription of genes related to proinflammatory cytokines.

RLRs are a family of receptors that include molecules of the RIG-I and MDA-5 (melanoma differentiation-associated gene-5) ${ }^{6}$. RIG-I and MDA- 5 display two $\mathrm{N}$-terminal caspase activation and recruitment domains (CARD domains) in tandem, a हैentral DExD/H-box domain consisting of two hẹlicase domains (Hel-1 and Hel-2), and a C-terminal'Fegulatory domain ${ }^{3,5,7}$. These receptors are cytoplasmic and detect viral RNA both single-stranded (ssRNA) and double-stranded (dsRNA).

CLRs contain three types of receptors. Type Ireceptors are transmembrane and contain several carbohydrate recognition domains (CRDs) (e.g. ED205 or DEC205, and macrophage mannose receptor 1 or 
MMR-1). Type II receptors are transmembrane proteins and typically contain only one CRD (e.g., DC-SIGN and Dectin-1 or -2). The third type has a soluble form and includes the mannose-binding lectin (MBL). In these CLRs, two conserved domains confer specificity. While EPN (Glu-Pro-Asn) motifs confer specificity to mannose, QPD (Gln-Pro-Asp) motifs have other CRDs ${ }^{8}$.

ALRs are cytoplasmic and express an $\mathrm{N}$-terminal pyrin domain (PYD) that binds to DNA. After binding, the PYD domain interacts with another PYD domain in the adapter protein ASC (apoptosis-associated speck-like protein that contains a CARD). Then, ASC recruits and activates procaspase 1 , and releases caspase- 1 that processes the inactive precursors of interleukin- $1 \beta$ (IL-1 $\beta$ ) into the mature form. This process induces an inflammatory form of cell death called pyroptosis ${ }^{5}$.

NLRs are receptors located mainly in the cytoplasm but also in mitochondria and express one of the four possible binding domains at the $\mathrm{N}$-terminal. According to these domains, we identify four subfamilies: (1) NLRA (A for acidic transactivating domain, e.g., CIITA), (2) NLRB (B for BIR, or baculovirus inhibitor of apoptosis protein repeat, e.g., NAIP), (3) NLRC (C for CARD, e.g., NOD2 or NLRC2), and (4) NLRP ( $P$ for PYD, e.g., NLRP3). In addition, these receptors contain an intermediate nucleotide-binding domain (NBD) also known as nucleotide oligomerization domain (NOD), which is necessary for binding and self-oligomerization. Finally, there is an LRR domain at the C-terminus ${ }^{8}$. The best-characterized function of NLRs is to sense PAMPs or DAMPs and activate the immune response.

\section{NLRs}

The family of NLRs consists of 22 receptors in humans and 34 in mice. In humans, only eight of these have been well-characterized (Fig. 2). NLRs express one of the three structural domains (CARD, PYD, or BIR motifs) at the $\mathrm{N}$-terminal which are involved in protein-protein interactions. Importantly, an intermediate NBD allows ATP binding and oligomerization. The C-terminal region in NLRs contains an LRR-domain similar to the one present in $\mathrm{TLRs}^{8}$. The activation of some NLRs, such as NLRC4, NLRP1, NLRP3, NLRP6, NLRP7, and NLRP12, stimulates the inflammasome, which is a multimeric protein complex that mediates the activation of inflammatory caspases ${ }^{9}$. Inflammasome activation leads the processing of caspase- 1 and -11 in mice and -4 and -5 in humans, which generates the active forms of IL-1 $\beta$, IL-18, and IL-33. Besides the processing of cytokines, these caspases contribute to the cleavage of gasdermin-D, which induces a particular type of cell death termed pyroptosis ${ }^{10}$.

Despite their central role in activating the imflammasome, NLRs have some other very importañt regulatory activities. For example, NOD2 and NLRP3 regulate the signaling pathways of innate immunity, including the canonical and non-canonical pathways of NF-kB, mitogen-activated protein kinase (M $\mathrm{M} A \mathrm{APK}$ ), and type I interferons (IFNs). They also regulate pathways that lead to the production of cytokines, chemokines, reactive oxygen species, and to the activation of ribonuclease $L^{11}$. NOD2 and NLRC 4 along with its correceptor NAIP5, control the induction of autophagy and mitophagy ${ }^{12}$. Meanwhile, NLRPIO and NLRC5 regulate and modulate another NLRs ormajor histocompatibility complex (MHC) genes through some molecules such as Class II, MHC, transẵctivator (CIITA $)^{11,13}$. Finally, NLRP2 and NLRP7 participate in embryonic/fetal development and uterine implantation, which are required to control the immune response ${ }^{14}$.

It is undeniable that NLRs and their multiprotein complexes participate in inflammasome-dependentas well as inflammasome-independent pathways. However, there is increasing evidence of their importance as the central effector of the immune response. A novel connection between endoplasmic reticulum stress and triggering of the innate immunity mediated by:NOD1 and NOD2 has been described ${ }^{15}$. The present review focuses on the functions of one NLR, the NOD2,during bacterial and viral infections, as well as on potymorphisms associated with disease susceptibility $\varepsilon_{\text {during }}$ infections and other pathogenic conditions. Moreover, the potential of NOD2 as a therapeutic targetio in the near future is exposed.

\section{NOD2 DURING BACTERIAL AND VIRĀL INFECTIONS}

The NOD2 receptor has a molecular weight of $110 \mathrm{kDa}$ (1040 aa) (Fig. 3). It expresses two CARD domains in the N-terminus, a central NBD, and an LRR 
Figure 2. NOD-like receptors (NLRs): General characteristics and functions for the eight best-characterized NLRs in humans. Some NLRs received a name according with the domain present in their amino-terminal, such as NLRCs that present a caspase-activation and recruitment domain (CARD) and NLRPs having with a Pyrin domain (PYD). Functions of these NLRs include the formation of inflammasome and nodosome (axis NOD2/RIP2), and some of them exhibit regulator activities.

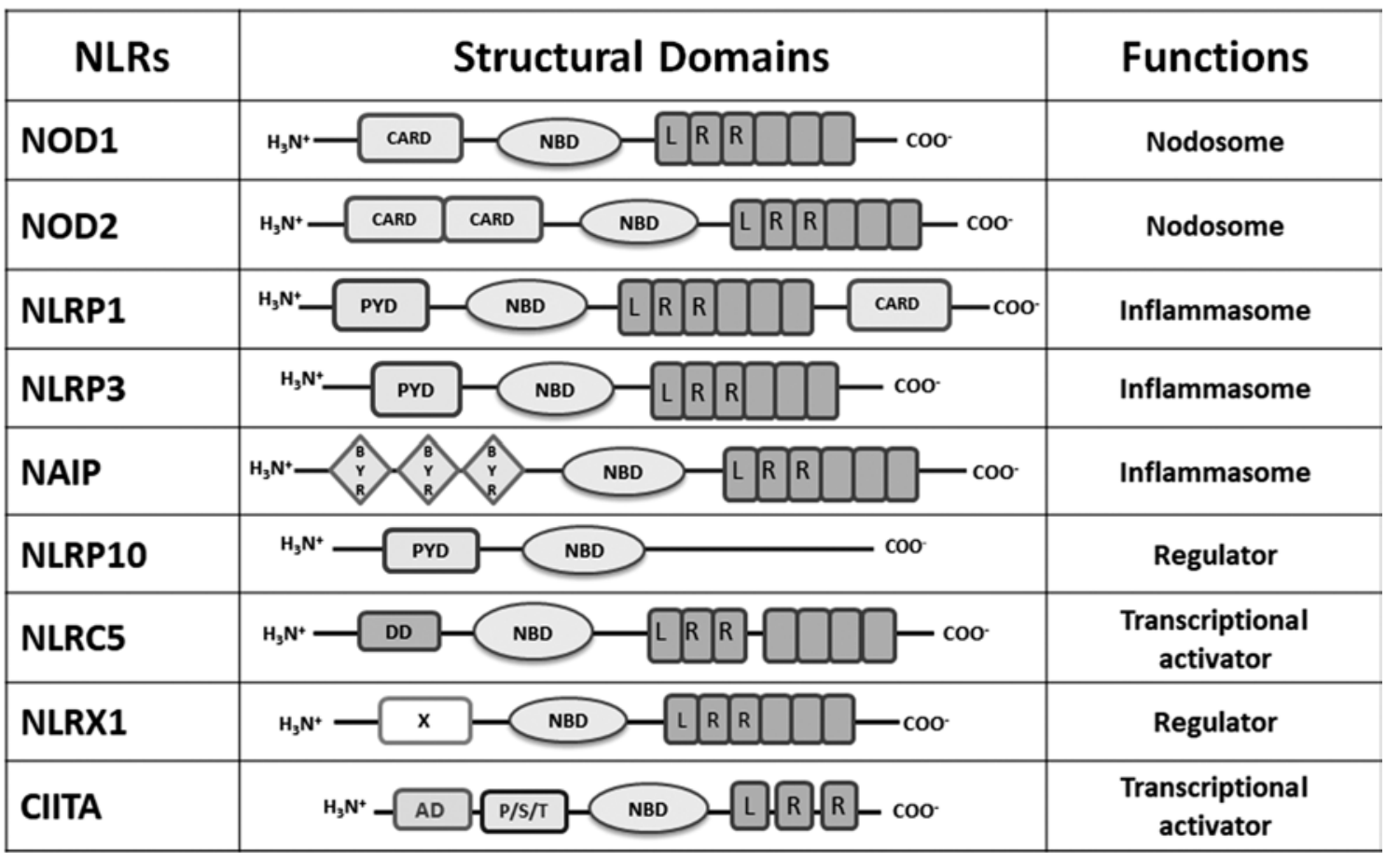

Figure 3. Protein interactions established by the cytoplasmic receptor NOD2 relevant for immune responses. NOD2 is a very dynamic receptor; once it is activated in the cells, numerous interactions and responses are mediated. NOD2 is involved inca broad range of cellular responses that include inflammasome regulation, production of proinflammatory cytokines, triggering autophagy, production of type I interferons, and other antiviral activities such the activation of RNAse L.

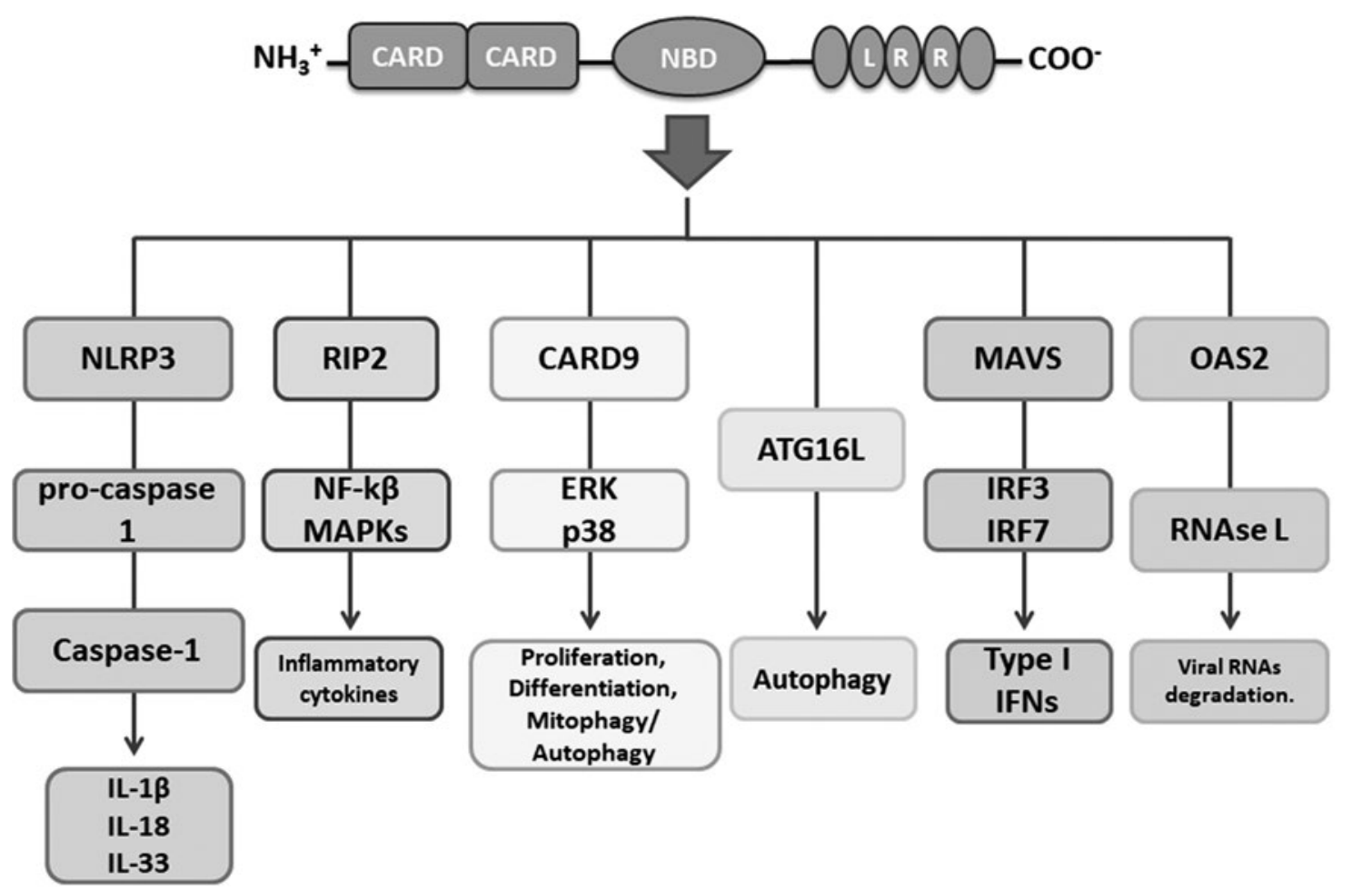


domain at the C-terminus ${ }^{16}$. NOD2 is able to detect muramyl dipeptide (MDP), a molecular motif commonly expressed in the peptidoglycan (PGN) of Gramnegative and Gram-positive bacteria. This receptor is commonly expressed in cells that include Paneth intestinal cells, monocytes/macrophages (Mo/M $\phi s$ ), dendritic cells (DCs), and granulocytes.

NOD2 is predominantly located in the cell cytoplasm and is able to interact with multiple proteins (Fig. 3). Following increased expression of protein in vitro, NOD2 becomes associated with the plasmatic membrane, an event that seems important to activate $\mathrm{NF}-\mathrm{KB}^{17}$. M $\phi$ s treated with MDP exhibit NOD2 molecule in their acidic vesicles ${ }^{1}$. Several studies on $M \phi s$ indicate that while DCs from animals and humans show that stimulation of NOD2 by MDP induces a variety of responses that clearly differ from those triggered by TLRs, the activation of NOD2 can, in fact, induce a two- to three-fold potentiation of the response mediated by TLRs $^{18}$.

NOD2 enables those TLRs localized in the cytoplasmic membrane to complete or increase their response. This is particularly important in infections caused by intracellular pathogens ${ }^{16}$. For example, after the recognition of MDP by NOD2, and LPS by TLR4, the activation of both PRRs is synergistic and cytokine production is significantly potentiated ${ }^{19}$. An analysis of transcripts from cells infected with vacuolar pathogens showed a TLR response dependent on induction of MyD88. Meanwhile, in cells infected with intracellular pathogens, a NOD2- and interferon regulatory factor 3 (IRF3)-dependent responses were found ${ }^{20}$.

Various authors have reported the involvement of NOD2 in bacterial infections (Fig. 4). NOD2 also participates in the expression of the inducible nitric oxide (NO) synthase to produce NO during Mycobacterium tuberculosis infections of human $\mathrm{M} \phi \mathrm{s}^{21}$. NOD2 mRNA levels increased in a rat model for Staphylococcus aureus-induced mastitis ${ }^{22}$, while NOD2 mRNA, as well as protein levels, increased in the central nervous system of a mouse model for pneumococcal meningitis ${ }^{23}$. NOD2 has also been reported to participate in the immune response to periodontal pathogens ${ }^{24}$ and contributes to the bone loss mediated by Porphyromonas gingivalis ${ }^{25}$. In cells infected with extracellular and intracellular bacteria, there is an increase in the expression and activation of NOD2, based on dependent and independent recruitment of receptor-interacting prötein 2 (RIP2). Apart from this significant interaction of NOD2 with RIP2, other non-canonical signaling pathwayys are also affected. Three of the most important interactions occurring with NOD2 during infections areherein described.

The response of NOD2 to MDP initiates a signaling cascade activating NF-KB and MAPK in a TLR-independent manner. Thus, NOD2 senses MDP through its LRR domain which then induces the unfolding of the NBD domain, followed by self-oligomerization, and exposure of its CARD domains ${ }^{1,16}$. These events Eead to the recruitment and binding of RIP226 by homophilic interactions through CARD-CARD ${ }^{27}$. The binding of NOD2 and RIP2 culminates in the activation of NF- $\mathrm{KB}$. RIP2 molecule recruits TNF receptor-associated factor 6-E3 ubiquitin-protein ligase, which subsequently triggers self-ubiquitination. The latter also has the-ability to polyubiquitinate other proteins downstream from $N O D 2^{28}$. The kinase domain of RIP2 is associated with other E3 ubiquitin ligases such as cellular inhibitors of apoptosis (cIAP1/2). Both catalyze the ubiquitination of RIP2 at the Lys63 site (K63). The polyubiquitination of RIP2 recruits the transforming growthfactor beta-activated kinase 1 complex (TAK1) GAK1$\mathrm{TAB} 1 / 2 / 3$ ), leading to the activation of the $\mathrm{IkK}$ kinases $^{29}$. The phosphorylation and recruitmentof the IkK $\beta$ kinase inhibit NF- $K B$ through degradation of IKBs by the proteasome $\mathrm{e}^{1,27}$ (Fig. 4).

CARD9 is expressed predominantly in DCs and M $\phi s$; consequently, it is found in lymphoid organs and is an adapter protein with a CARD domain at the $\mathrm{N}$-terminus and a "coiled-coil" domain at the C-terminus. lates signaling during fungal infections and is required in the immune response against intracellular pathogens $^{30}$. In M $\phi s$ and DCs treated with PGN, ENOD2 interacts with CARD9; this interaction activates p38 extracellular signal-regulated kinase $(E R K)^{1}$ and c-Jun $\mathrm{N}$-terminal kinase (JNK), which activates the heterodimeric transcription factor activator protein $1^{29}$. The M $\phi s$ of mice deficient in Card9-/- express. defects in the activation of p38 and JNK kinases after a viral and bacterial infection, but not in NF-kB. Whereas the overexpression of CARD9 and NOD2 normally occurs during infection with Listeria monocytoggenes, in CARD9-/- mice cytokine production is deficient, and this bacterium cannot be eliminated ${ }^{30}$. 
Figure 4. NOD2-activated pathways during infections with extracellular and intracellular bacteria. This figure shows a general overview of interactions established by NOD2 through its structural domains. (1) The canonical pathway depends on NOD2 interaction with RIP2 that leads downstream activation of NF-kB. (2) A non-canonical pathway on NOD2 and CARD9 leads to the activation of AP-1. (3) Another non-canonical pathway that NOD2 activates involves the interaction with the ATG16L 9 rotein. A final effect of signaling through these pathways is the production of proinflammatory cytokines and autophagy. RIP2: Receptorinteracting protein 2, NF- $\mathrm{kB}$ : Nuclear factor $\kappa \mathrm{B}, \mathrm{CARD}$ : Caspase activation and recruitment domains, AP-1: Activator protein 1 .

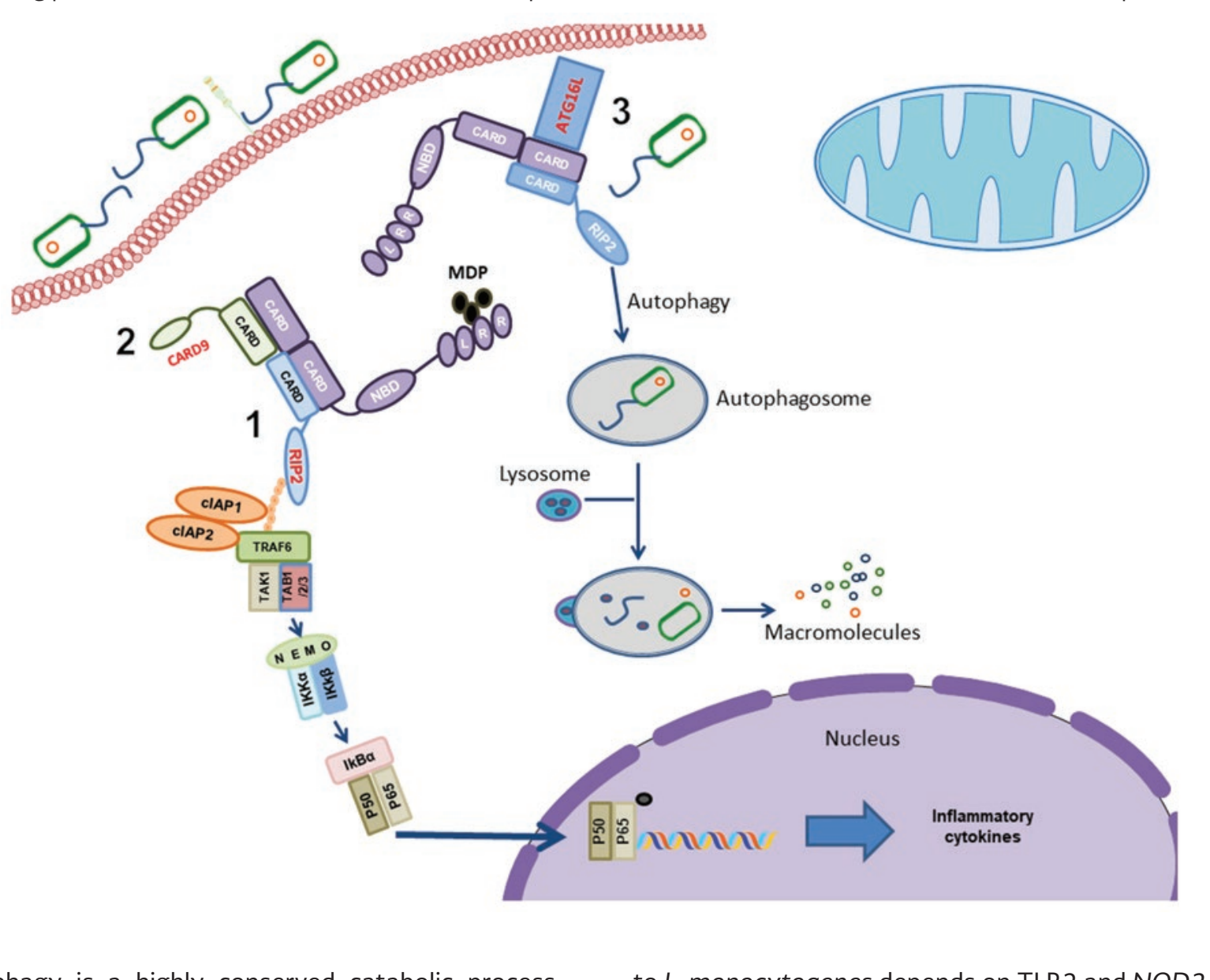

Autophagy is a highly conserved catabolic process of eukaryotic cells used to recycle macromolecules ${ }^{26}$. This occurs in double-membrane vesicles that sequester damaged organelles, which are degraded afterward by the fusion of lysosomes to these vesicles. Membrane-recruited NOD2 participates in the formation of autophagosome by interacting with the autophagy-related 16-like 1 protein complex (ATG16L1). During the bacterial invasion, ATG16L1 and NOD2 lead bacteria elimination. Although it is known to be an NF-kB-independent mechanism, the involvement of RIP2 is still being debated ${ }^{28,31}$ (Fig. 4). The M $\phi$ s of $\mathrm{TLR2}^{-/-}, \mathrm{NOD}^{-/-}$, and RIP2 ${ }^{-1--}$ mice are more susceptible to the infection with $L$. monocytogenes. In this infection model, the activation of the axis NOD2/ RIP2 and the consequent induction of autophagy is dependent on the ATG16L protein, and the ERKsignaling pathway ${ }^{31,32}$. Thus, the protective response to L. monocytogenes depends on TLR2 and NOD̈2 signaling. In infections with Salmonella enterica Serovar typhimurium, the induction of autophagy by NOD2 is necessary for the antigen presentation to take place in $\mathrm{DCs}^{33}$.

NOD2 stimulation with viral ssRNA activates the interferon regulatory factors 3 and 7 (IRF3 and IRF7) and induces an antiviral response mediated by type I IFNs. This has raised new questions about molecular recognition and signaling by NOD2 because thêre are no structural similarities between bacterial MDP and viral ssRNA or dsRNA motifs ${ }^{34}$. NOD2 along wit other NLRs, such as NOD1, might promote inflammation and facilitate antiviral response ${ }^{35}$. It is plausibte that the activation of NOD2 occurs following a direct interaction with viral genome or proteins during the viral replication cycle $^{36}$. 
Figure 5. NOD2 activation during viral infections. Distinct pathways involving NOD2 are triggered by viruses and include: (1) The canonical MDP activation pathway involving NOD2 and the kinase RIP2 with the subsequent activation of NF- $\mathrm{BB}$, leading to inflammatory gene expression, (2) The second pathway involves ssRNA virus-induced activation of NOD2, which binds tô-MAVS, leading to IRF3/IRF7 activation and interferon gene expression, and (3) Another non-canonical pathway involves NOD2 and OAS2 interaction which activates RNAse $L$ that degrades viral RNA. MDP: Muramyl dipeptide, RIP2: Receptor-interacting protein 2 NF- $\kappa$ B: Nuclear factor $\kappa B$, MAVS: Mitochondrial antiviral-signaling protein, IRF: Interferon regulatory factors, OAS2: 2'-5'-oligoadenylate synthetase type 2 .

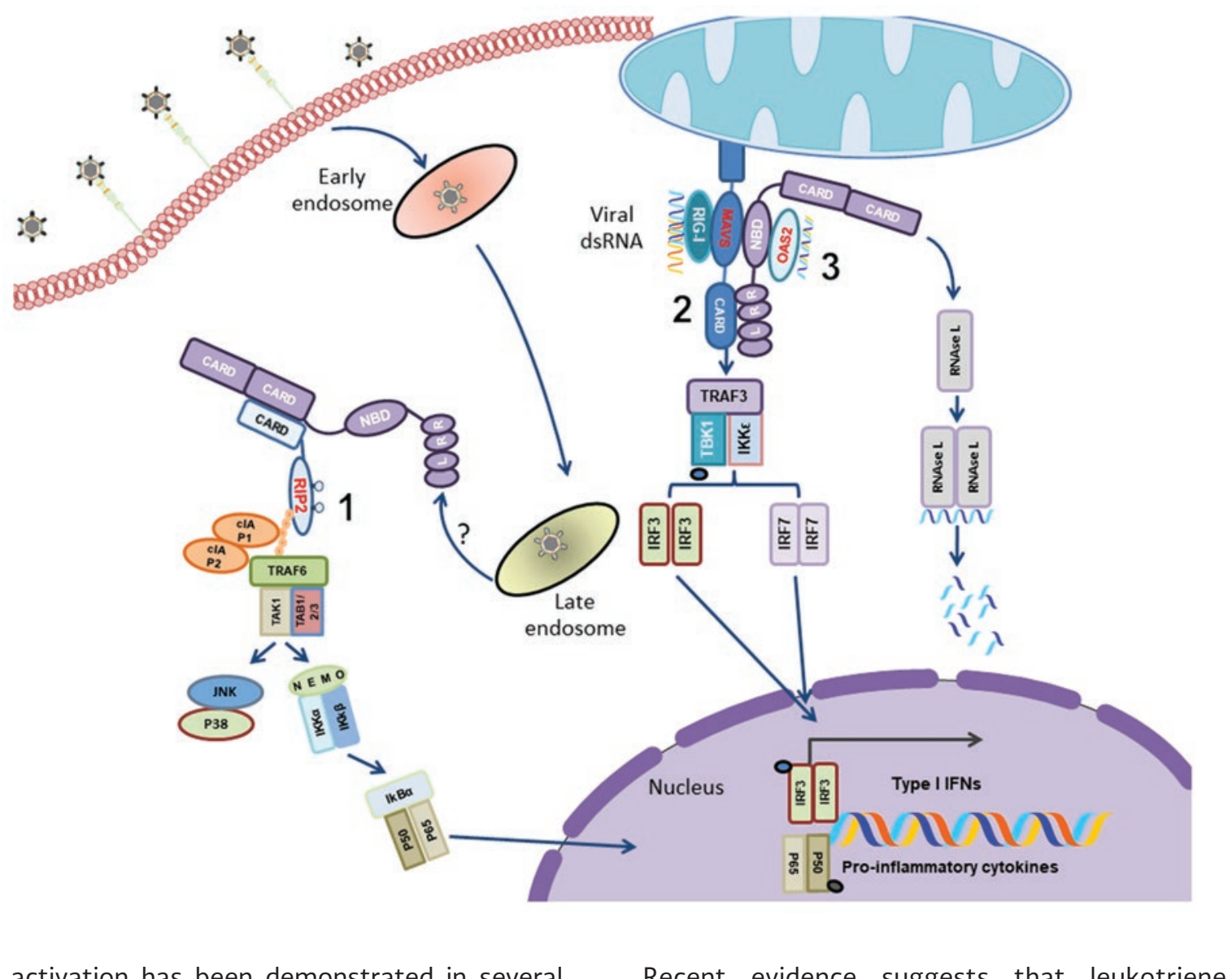

NOD2 activation has been demonstrated in several infections with viruses (Fig. 5) that have an ssRNA genome such as vesicular stomatitis virus, respiratory syncytial virus (RSV), and parainfluenza virus 3, and also for some viruses with DNA genomes such as human cytomegalovirus. In these infections, there is an increased expression of NOD2 and type I IFNs in human bronchial epithelial cells, M $\phi s$, and embryonic fibroblasts ${ }^{36,37}$. In M $\phi s$ and epithelial cells NOD2 binds to other proteins apart from RIP2 including CARD9, ATG16L, mitochondrial antiviral-signaling protein (MAVS), and 2'-5'-oligoadenylate synthetase type 2 $(\text { OAS2) })^{16}$ (Fig. 5).

A study of neutrophils from individuals exposed to human immunodeficiency virus revealed that the hyporesponse of NOD2 and other effectors are related to the maintenance of seronegativity ${ }^{38}$.
Recent evidence suggests that leukotriene B4 directly impacts on the NOD2 pathway enhancing the immune response against influenza A virus (IAV) ${ }^{39}$. On the other hand, hepatitis $E$ virus exhibits anintrinsic ability to counteract the activity of NOD2 and other PRRs ${ }^{40}$.

During viral infections, an activation of the Eanonic axis NOD2/RIP2 also occurs. In an infection model of $\mathrm{NOD}^{-/-}$and RIP2 ${ }^{-/-}$mice with IAV, these become hypersensitive to the infection. The analysis of individual of RIP2-/- cells showed that this is dueto the induction of mitophagy (mitochondrial autophagy), a phenomenon that causes an increase in csuperoxide production and leads to mitochondrial damage. This causes a strong activation of the NLRP3 inflammasome, resulting in increased production of IL-18. The RIP2 protein regulates mitophagy through 
phosphorylation of the Unc-51-like kinase 1 . This model demonstrates that NOD2 and RIP2 downregulate the activation of the NLRP3 inflammasome and production of IL-18 through ULK-1 ${ }^{41}$.

In addition, during viral infections, NOD2 can also be relocated to the mitochondria by its interaction with the MAVS protein through its LRR and NOD domains. This interaction triggers the nuclear translocation of IRF 3 and induces the production of type I IFNs ${ }^{26,28}$ (Fig. 5). The activation mechanism involves macromolecular complexes consisting of RIP2 and TRAF3 ${ }^{28,29}$. In fact, depletion of NOD2 abates the expression of type I IFNs. Viral ssRNAs may activate the NOD2 receptor, causing activation of the MAVS protein in the mitochondria. MAVS phosphorylates IRF3/IRF7, two factors that form homodimers and translocate to the nucleus ${ }^{26,36}$. Moreover, the interaction of NOD2 and the adapter protein MAVS regulate the production of type I IFN and increase expression of NOD2 de novo. It is possible that NOD2 interacts with the MAVS-RIG-I/ MDA-5 complex associated with viral RNA ${ }^{11}$.

In a murine model of infection with RSV, the activation of NOD2 allows its relocation to the mitochondria and increases NOD2 mRNA expression coinciding with the increased expression of RIG-I and TLR3, suggesting the synergic role of the latter two receptors in the antiviral response ${ }^{37}$. Proteomic analysis revealed that NOD2 interacts with OAS2 in the THP-1 cell line ${ }^{42}$. The OAS2 molecule is necessary for the activation of RNase $L$, which degrades viral and cellular RNA limiting viral replication ${ }^{14,51}$. The interaction between NOD2 and OAS2 takes place during cellular response to dsRNA and synthetic ligands such as poly $(\mathrm{I}: \mathrm{C})$, which mimic viral RNAs. Data suggest that in some viral infections, NOD2 possibly participates in inducing the expression of type I IFNs through a mechanism independent of $\mathrm{RLRs}^{26}$. Clearly, NOD2 is a dynamic cytoplasmic receptor exhibiting an exceptional plasticity, whose cellular activity depends on the adapter molecules recruited in each response ${ }^{36,37}$.

\section{NOD2 POLYMORPHISMS INVOLVED IN HEALTH AND DISEASE}

NOD2 deficiency in mice has been associated with chronic inflammatory disease. In humans, a mutation in the CARD15 gene (in the LRR region of NOD2) has been associated with chronic bowel inflammation in Crohn's disease (CD). Moreover, polymorphisms in this receptor are associated with various diseases such as Blau syndrome, arthritis, atopic dermatitis, șarcoidosis, and possibly asthma ${ }^{43,44}$.

The gene encoding NOD2 is located in the "q"चregion of chromosome 16 and is highly polymorphic (Fig. 6), with at least 660 single nucleotide polymorphisms (SNPs) described with alleles varying among individual populations and across geographical locations. There are three main polymorphisms in NOD2 frequently associated with disease, SNP8 (rs2066844), SNP12 (rs2066845), and SNP13 (rs414500 53$)^{45}$. Individuals that are heterozygous for any of these SNPs show a 2- to 4-fold greater risk of developing $C D$, while those homozygous for these SNPs exhibit an almost 20-fold greater risk of developing this disease ${ }^{46,47}$

SNPs 8,12 , and 13 in the NOD2 gene are located in exons 4,8 , and 11 , respectively. Whereas there is only an amino acid change in SNP8 and 12, a frameshift takes place in SNP13, leading to the emergence of a truncated protein ${ }^{48}$. Some polymorphisms in the region coding for NOD2/CARD15 increase the risk of developing $C D$ up to 17.1-fold in either homozygous or heterozygous individuals ${ }^{49}$. Mutations in NOD2/ CARD15 decrease activity in the pathway, Peading to inhibition of NF-KB. This causes the over-reactivation of this protein complex and the proinflammatory symptoms observed in $\mathrm{CD}^{50}$.

In addition to $C D$, these polymorphisms in NOD2 have been implicated in other ailments such as anquilosante spondylitis, arthritis, and cancer ${ }^{50}$. Moreover, NOD2 (rs8057431) seems to be implicated in susceptibility to Mycobacterium leprae in diverse popylations ${ }^{51}$. Furthermore, the SNP13 in NOD2 has been implicated in septic shock following transplantation wit stem cells ${ }^{52}$.

Strikingly, NOD2 sensing activity of commensâls has been shown to be indispensable to aid in the right sorting of antimicrobial peptides to the intracellular compartments of the Paneth cells and also for the eorrect establishment of symbiosis ${ }^{53}$. Thus, in CD assöclated with NOD2 deficiencies, the ability to respond to the MDP present in commensal and pathogenic bacteria is clearly altered ${ }^{54}$. 
Figure 6. Chromosomal location, gene, and protein characteristics for nod2 and NOD2. The gene encoding for NOD2 protein is located in the $\mathrm{q}$ arm of chromosome 16. The gene is constituted by multiple exons and introns distributed along $39.5 \mathrm{~kb}$. The protein contains 1040 aa.

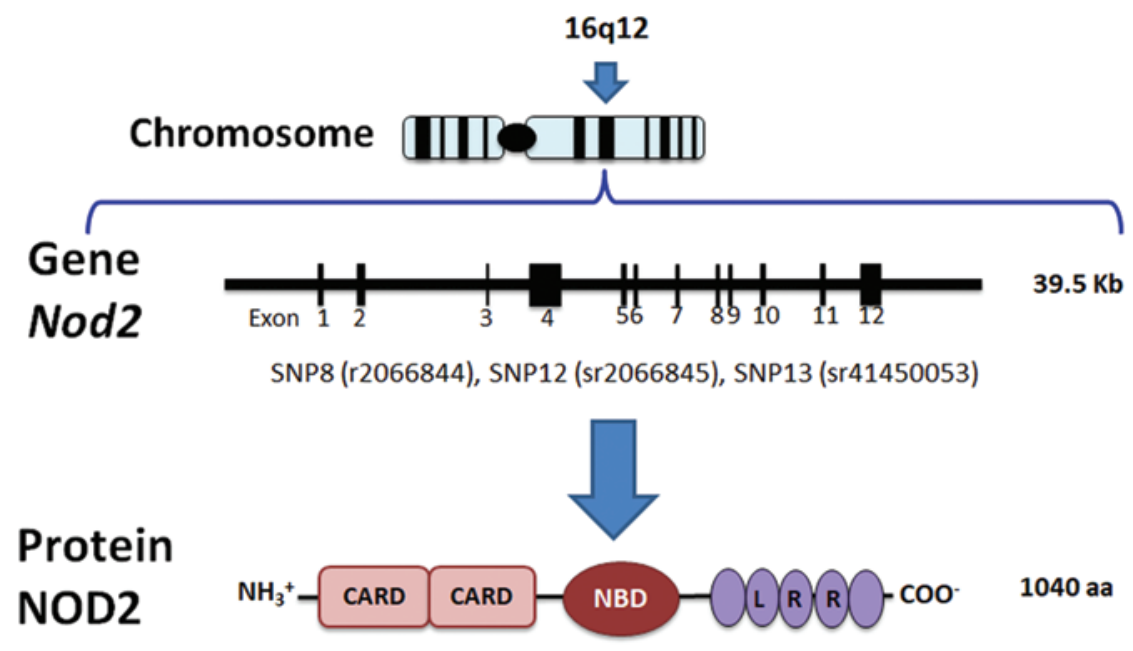

\section{IS NOD2 A SUITABLE THERAPEUTIC TARGET?}

Current data on NLRs suggest that these molecules hold a central role in the innate immune response as well as in regulating proinflammatory pathways. In 2008, for example, two independent studies reported that NLRP3 is a chief sensor in response to aluminum salts 55,56 , which are the lone approved adjuvant for human vaccines. This represents the first insight into the action mechanisms for these salts. Stimulation of NOD2 by MDP triggers a wide repertoire of transcripts in vitro, including those encoding for chemokines, proinflammatory cytokines, antimicrobial peptides, and adhesion molecules ${ }^{57}$. Several authors have demonstrated that resistance to viruses is conferred by MDP alone or in combination with other agents. As MDP signaling is mostly carried out through NOD2, this NLR has been envisioned as a potential therapeutic target ${ }^{58}$.

Considering that NOD2-mediated inflammation exacerbates some ailments, it could be a suitable therapeutic target through its downregulation. An important point of intervention is through the RIP2 molecule, which as mentioned is downstream of the NOD2 signaling pathway. RIP2 activity can be effectively impeded by existing pharmaceutical type II kinase inhibitors ${ }^{59}$.

\section{CONCLUDING REMARKS}

NOD2, a PRR, exhibits critical activities during bacterial and viral infections. NOD2 is able to synergize with TLRs and has an important role in the activation of NF-kB. Moreover, due to its dynamic nature, NOD2 can determine multiple cell responses not only in infectious diseases but also in health. Therefore, this molecule constitutes an important target for pathogen evasion mechanisms and is also a potential target to be intervened therapeutically in the future.

\section{ACKNOWLEDGMENTS}

The authors thank Allen Larsen and Jesús M. बجTorres Flores for critical and helpful comments an this manuscript.

\section{REFERENCES}

1. Shaw MH, Reimer T, Kim YG, Nuñez G. NOD-like receptors (NLRs): Bona fide intracellular microbial sensors. Gürr Opin Immunol. 2008;20:377-82.

2. Krishnaswamy JK, Chu T, Eisenbarth SC. Beyond pattern recognition: NOD-like receptors in dendritic cells. Trends Jmmunol. 2013;34:224-33.

3. Takeuchi $\mathrm{O}$, Akira $\mathrm{S}$. Pattern recognition receptors and inflammation. Cell. 2010;140:805-20.

4. Akira S, Takeda K. Toll-like receptor signalling. Nat Rev Immunol. 2004;4:499-511. 
5. Wu J, Chen ZJ. Innate immune sensing and signaling of cytosolic nucleic acids. Annu Rev Immunol. 2014;32:461-88.

6. Yoneyama M, Kikuchi M, Matsumoto K, et al. Shared and unique functions of the DExD/H-box helicases RIG-I, MDA5, and LGP2 in antiviral innate immunity. J Immunol. 2005;175:2851-8.

7. Kolakofsky D, Kowalinski E, Cusack S. A structure-based model of RIG-I activation. RNA. 2012;18:2118-27.

8. Kanneganti T. Central roles of NLRs and inflammasomes in viral infection. Nat Rev Immunol. 2010;10:688-98.

9. Latz E, Xiao TS, Stutz A. Activation and regulation of the inflammasomes. Nat Rev Immunol. 2013;13:397-411.

10. Shi J, Zhao Y, Wang K, et al. Cleavage of GSDMD by inflammatory caspases determines pyroptotic cell death. Nature. 2015;526:660-5.

11. Ting J, Duncan J, Lei Y. How the noninflammasome NLRs function in the innate immune system. Science. 2010;327: 286-90.

12. Byrne BG, Dubuisson JF, Joshi AD, Persson JJ, Swanson MS. Inflammasome components coordinate autophagy and pyroptosis as macrophage responses to infection. MBio. 2013;4: e00620-12.

13. Lamkanfi M, Kanneganti TD. Regulation of immune pathways by the NOD-like receptor NLRC5. Immunobiology. 2012; 217:13-6.

14. Lupfer C, Kanneganti T. Unsolved mysteries in NLR biology. Front Immunol. 2013;4:285.

15. Keestra-Gounder AM, Byndloss MX, Seyffert N, et al. NOD1 and NOD2 signalling links ER stress with inflammation. Nature. 2016:532:394-7.

16. Strober W, Watanabe T. NOD2, an intracellular innate immune sensor involved in host defense and crohn's disease. Mucosal Immunol. 2011:4:484-95.

17. Lécine P, Esmiol S, Métais JY, et al. The NOD2-RICK complex signals from the plasma membrane. J Biol Chem 2007;282: 15197-207.

18. Fritz JH, Girardin SE, Fitting C, et al. Synergistic stimulation of human monocytes and dendritic cells by toll-like receptor 4 and NOD1-and NOD2-activating agonists. Eur J Immunol. 2005;35:2459-70

19. Travassos LH, Girardin SE, Philpott DJ, et al. Toll-like receptor 2-dependent bacterial sensing does not occur via peptidoglycan recognition. EMBO Rep. 2004;5:1000-6.

20. Leber JH, Crimmins GT, Raghavan S, et al. Distinct TLR- and NLR-mediated transcriptional responses to an intracellular pathogen. PLoS Pathog. 2008;4:e6.

21. Landes MB, Rajaram MV, Nguyen H, Schlesinger LS. Role for NOD2 in Mycobacterium tuberculosis-induced iNOS expression and no production in human macrophages. J Leukoc Biol. 2015;97:1111-9.

22. Wang H, Yu G, Yu H, et al. Characterization of TLR2, NOD2, and related cytokines in mammary glands infected by Staphylococcus aureus in a rat model. Acta Vet Scand. 2015;57:25.

23. Liu X, Han Q, Leng J. Analysis of nucleotide-binding domain oligomerization domain proteins in a murine model of pneumococcal meningitis. BMC Infect Dis. 2014;14:648.

24. Marchesan J, Jiao Y, Schaff RA, et al. TLR4, NOD1 and NOD2 mediate immune recognition of putative newly identified periodontal pathogens. Mol Oral Microbiol. 2016;31:243-58.

25. Prates TP, Taira TM, Holanda MC, et al. NOD2 contributes to Porphyromonas gingivalis-induced bone resorption. J Dent Res 2014;93:1155-62

26. Lecat A, Piette J, Legrand-Poels S. The protein nod2: An innate receptor more complex than previously assumed. Biochem Pharmacol. 2010;80:2021-31.

27. Hasegawa M, Fujimoto Y, Lucas PC, et al. A critical role of RICK/ RIP2 polyubiquitination in nod-induced NF-kappaB activation. EMBO J. 2008:27:373-83

28. Jakopin $\breve{Z}$. Nucleotide-binding oligomerization domain (NOD) inhibitors: A rational approach toward inhibition of NOD signaling pathway. J Med Chem. 2014;57:6897-918.
29. Wertz IE, Dixit VM. Signaling to NF-kappaB: Regulation by ubiquitination. Cold Spring Harb Perspect Biol. $2010 ; 2$ a003350

30. Hsu YM, Zhang Y, You Y, et al. The adaptor protein CARD9 is required for innate immune responses to intracellular pathogens. Nat Immunol. 2007;8:198-205.

31. Travassos LH, Carneiro LA, Ramjeet M, et al. Nodl and nod2 direct autophagy by recruiting ATG16L1 to the plasma membrane at the site of bacterial entry. Nat Immunot. 2010; 11:55-62.

32. Anand PK, Tait SW, Lamkanfi M, et al. TLR2 and RIP2 $\overline{\text { pathways }}$ mediate autophagy of Listeria monocytogenes via extracellular signal-regulated kinase (ERK) activation. J Bio 2011:286:42981-91.

33. Cooney R, Baker J, Brain O, et al. NOD2 stimulation induces autophagy in dendritic cells influencing bacterial handling and antigen presentation. Nat Med. 2010;16:90-7.

34. Monie TP. NLR activation takes a direct route. Trends $\overline{B i o c h e m}$ Sci. 2013;38:131-9.

35. Coutermarsh-Ott S, Eden K, Allen IC. Beyond the inflammasome: Regulatory NOD-like receptor modulation of the host immune response following virus exposure. J Gen Virob 2016; 97:825-38.

36. Sabbah A, Chang TH, Harnack R, et al. Activation of innate immune antiviral responses by nod2. Nat . Immunol. 2009;10:1073-80

37. Vissers $M$, Remijn $T$, Oosting $M$, et al. Respiratory syncytial virus infection augments NOD2 signaling in an IFN- $\beta$-dependent manner in human primary cells. Eur J Immunol. 2012;42: 2727-35

38. Hernandez J, Giraldo D, Paul S, Urcuqui-Inchima S. Invølvement of neutrophil hyporesponse and the role of toll-like receptors in human immunodeficiency virus 1 protection. PLOS One. 2015;10:e0119844.

39. Le Bel M, Gosselin J. Leukotriene B4 enhances NOD2-dependent innate response against influenza virus infection. PLoS One. 2015; 10:e0139856.

40. Lei Q, Li L, Cai J, et al. ORF3 of hepatitis E virus inhibits the expression of proinflammatory cytokines and chemotactic factors in LPS-stimulated human PMA-THP1 cells by $\overline{\text { phibiting }}$ NF-kB pathway. Viral Immunol. 2016;29:105-11.

41. Lupfer $C$, Thomas $P$, Anand $P$, et al. Receptor interacting protein kinase 2-mediated mitophagy regulates inflammasome activation during virus infection. Nat Immunol. 2013;14:480-8.

42. Lupfer $\mathrm{C}$, Thomas $\mathrm{P}$, Kanneganti T. Nucleotide oligomerization and binding domain 2-dependent dendritic cell activation is necessary for innate immunity and optimal $C D 8^{+} \mathrm{T}$ Cell responses to influenza a virus infection. J Virol. 2014;88:8946-55.

43. Kobayashi KS, Chamaillard M, Ogura Y, et al. Nod2-dependent regulation of innate and adaptive immunity in the intestinal tract. Science. 2005;307:731-4.

44. Moreira LO, Zamboni DS. NOD1 and NOD2 signaling in infection and inflammation. Front Immunol. 2012;3:328.

45. Jaskula $E$, Lange $A$, Kyrcz-Krzemien S, Markiewicz $M$, Dzierzak-Mietla M, Jedrzejczak WW, et al. NOD2/CARD1 5 single nucleotide polymorphism 13 (3020insC) is associated with risk of sepsis and single nucleotide polymorphism 8 (2I04C $>\mathrm{T}$ ) with herpes viruses reactivation in patients after allogeneic hematopoietic stem cell transplantation. Biol BloodoMarrow Transplant. 2014;20:409-14

46. Tello-Ruiz M, Walsh E, Rioux J. Gastroenterologic andhepatic disease. Madame Curie Bioscience Database. Austin (TX): Lande Bioscience; 2000

47. Cantó E, Ricart E, Busquets $D$, et al. Influence of a nucleotide oligomerization domain 1 (NOD1) polymorphim and NOD2 mutant alleles on crohn's disease phenotype. World J Gastroenterol 2007:13:5446-53.

48. Chamaillard M, Philpott D, Girardin SE, et al. Gene-environment interaction modulated by allelic heterogeneity in inflammatory diseases. Proc Natl Acad Sci U S A. 2003;100:3455-60. 
49. Strober W, Asano N, Fuss I, Kitani A, Watanabe T. Cellular and molecular mechanisms underlying NOD2 risk-associated polymorphisms in crohn's disease. Immunol Rev. 2014;260: 249-60.

50. Inohara N, Nuñez G. NODs: Intracellular proteins involved in inflammation and apoptosis. Nat Rev Immunol. 2003;3:371-82.

51. Sales-Marques C, Salomão H, Fava VM, et al. NOD2 and CCDC122-LACC1 genes are associated with leprosy susceptibility in Brazilians. Hum Genet. 2014;133:1525-32.

52. Grube M, Brenmoehl J, Rogler G, Hahn J, Herr W, Holler E. Donor Nucleotide-Binding oligomerization-containing protein 2 (NOD2) single nucleotide polymorphism 13 is associated with septic shock after allogenic stem cell transplantation. Biol Blood Marrow Transplant. 2015;21:1399-404.

53. Zhang Q, Pan Y, Yan R, et al. Commensal bacteria direct selective cargo sorting to promote symbiosis. Nat Immunol. 2015;16: 918-26.

54. Salem M, Seidelin JB, Eickhardt S, et al. Species-specific engagement of human nucleotide oligomerization domain 2 (NOD)2 and toll-like receptor (TLR) signalling upon intracellularbacterial infection: Role of crohn's associated NOD2 gene variants. Clin Exp Immunol. 2015;179:426-34.

55. Franchi L, Núñez G. The nlrp3 inflammasome is criticât for aluminium hydroxide-mediated IL-1beta secretion but dispensable for adjuvant activity. Eur J Immunol. 2008;38:2085-90

56. Eisenbarth SC, Colegio OR, O'Connor W, Sutterwala FS Flavell RA. Crucial role for the nalp3 inflammasome in the immunostimulatory properties of aluminium adjuvants. Nature. 2008;453:1122-6.

57. Geddes K, Magalhães JG, Girardin SE. Unleashing the therapeutic potential of NOD-like receptors. Nat Rev Drug Discov. 2009;8:465-79.

58. Kool M, Soullié T, van Nimwegen $M$, et al. Alum adjuvant boosts adaptive immunity by inducing uric acid and activating inflammatory dendritic cells. J Exp Med. 2008;205:869-82. 0

59. Canning P, Ruan Q, Schwerd T, et al. Inflammatory signaling by NOD-RIPK2 is inhibited by clinically relevant type II kinase inhibitors. Chem Biol. 2015;22:1174-84. 